\title{
A CROSS-SECTIONAL ANALYSIS OF DERMOSCOPIC PATTERNS DISTINGUISHING BETWEEN PSORIASIS AND LICHEN PLANUS: A STUDY OF 80 PATIENTS
}

\author{
Penmetcha Lakshmi Chandravathi' ${ }^{1}$ Praneet Awake ${ }^{2}$, Madhavi Kota ${ }^{3}$ \\ 1 Professor and HOD, Department of Dermatology, Venereology and Leprosy, Care Institute of Medical Sciences, Hyderabad, Telangana. \\ ${ }^{2}$ Post Graduate Student, Department of Dermatology, Venereology and Leprosy, Care Institute of Medical Sciences, Hyderabad, \\ Telangana. \\ ${ }^{3}$ Post Graduate Student, Department of Dermatology, Venereology and Leprosy, Care Institute of Medical Sciences, Hyderabad, \\ Telangana.
}

\section{ABSTRACT}

\section{BACKGROUND}

Dermoscope is non-invasive diagnostic tool, which allows rapid and magnified in-vivo observation of the skin and helps in visualization of morphologic features invisible to naked eye. Dermoscopy is useful in evaluating pigmented lesions, but it can also be used in evaluating the inflammatory skin disorders but little is currently known about their dermoscopic features. Dermoscopy of Psoriasis shows red globules, red lines, red dots, glomeruli-like vessels and light red background. Dermoscopy of Lichen planus shows Wickham's striae, central yellow brown area, grey blue dots, comedo-like opening, corn pearls.

\begin{abstract}
AIMS
To determine and compare dermoscopic patterns of Psoriasis and Lichen Planus and correlate the dermatoscopic images with histopathology of Psoriasis and Lichen planus in clinically difficult cases and compare the findings seen in our study with previous studies.

\section{MATERIALS AND METHODS}

A cross-sectional comparative study, during course of 2 years, total of 80 cases in which 40 patients each of Psoriasis and Lichen planus were included. Digital dermoscopic images of lesions were obtained with Hanse microscope, after lesions were covered with olive oil. All dermoscopic findings of both psoriasis and lichen planus were observed, captured and data was analysed statistically. In clinically difficult and atypical cases, histopathology was done and correlated with dermoscopy.
\end{abstract}

\section{STATISTICAL ANALYSIS}

For all the statistical analysis SPSS statistical software, version 17.0 for windows (SPSS Inc. Chicago, IL, USA) was used. ChiSquare $\left(\chi^{2}\right)$ test was done for statistical significance. In all instances, a "p" value of 0.05 was considered significant. Charts were prepared with Microsoft Excel 2010 version.

\section{RESULTS}

On dermoscopy there is statistical significance at $5 \%$ confidence interval of red globules $\left(p=0.001, \chi^{2}=37.143\right)$, Wickham's striae $\left(\mathrm{p}=0.001, \chi^{2}=34.286\right)$, grey blue dots $\left(\mathrm{p}=0.000, \chi^{2}=13.067\right)$ and glomeruli like vessels $\left(\mathrm{p}=0.003, \chi^{2}=8.538\right)$ in comparison between psoriasis and lichen planus. Dermoscopic findings of psoriasis were predominantly vascular while those of lichen planus were predominantly non-vascular.

\section{CONCLUSION}

Clinical use of dermoscopy in inflammatory dermatosis improves diagnostic ability and improves fundamental aspects of daily practice such as improvement of morphologic knowledge for visual tele-dermatology and in addition plays a psychological placebo effect on patients suffering from common inflammatory dermatosis.

\section{KEYWORDS}

Dermoscopy, Red Globules, Wickham's Striae, Psoriasis, Lichen Planus.

HOW TO CITE THIS ARTICLE: Chandravathi PL, Awake P, Kota M. "A cross-sectional analysis of dermoscopic patterns distinguishing between psoriasis and Lichen planus: A study of 80 patients." Journal of Evolution of Medical and Dental Sciences 2015; Vol. 4, Issue 105, December 31; Page: 17017-17022, DOI: 10.14260/jemds/2015/2574

\section{INTRODUCTION}

Skin surface microscopy was started hundreds of years ago, first in 1663 by Kolhaus. In 1920, Goldman coined the term "Dermoscopy" and showed its use to evaluate pigmented cutaneous lesions.

Financial or Other, Competing Interest: None.

Submission 02-12-2015, Peer Review 03-12-2015,

Acceptance 26-12-2015, Published 30-12-2015.

Corresponding Author:

Dr. Praneet Awake,

Department of Dermatology,

Care Hospital, Road No. 10, Banjara Hills,

Hyderabad-500034,

Telangana, India.

E-mail: awakepraneet@gmail.com

DOI:10.14260/jemds/2015/2574
Psoriasis and lichen planus are common inflammatory skin diseases and their characteristic appearance allows a clinical diagnosis in a high proportion of patients. However, unusual presentations at times do exist and may cause difficulties in the differentiation among these entities. In those cases, histopathology contributes significantly to the accurate diagnosis.

Dermoscope is non-invasive diagnostic tool, which allows rapid and magnified in-vivo observation of the skin and helps in visualization of certain morphologic features invisible to naked eye. It has also been called 'skin surface microscope', 'epiluminence microscope' or 'episcope.'1 It works on principal of "transillumination" of lesion and studying it with high magnification to visualize subtle features. ${ }^{2}$ Thus it forms a link between macroscopic clinical dermatology and microscopic dermatopathology. ${ }^{3}$ This "sub-macroscopic" observation of 
colours and structures enhances clinical assessment by providing new diagnostic criteria for the differentiation.

Dermoscopic patterns are seen in many common inflammatory dermatoses. Psoriasis vulgaris presents with vascular changes like dotted vessels, red dots and light red background and lichen planus presents with characteristic Wikham's striae, grey-blue background on dermoscopy.3,4 Dermoscopy, besides helping in the diagnosis, can be used to monitor treatment response. Given that Plaque Psoriasis and other inflammatory skin diseases may sometimes be difficult to differentiate clinically, a more detailed determination of specific dermoscopic patterns of inflammatory skin diseases could be a valuable addition for the clinical assessment. ${ }^{5-9}$

Purpose of this study is to analyse various dermoscopic patterns of psoriasis and lichen planus. With the acceptance of standardized dermoscopic criteria worldwide as diagnostic test would obviate the need for invasive histopathology.

\section{MATERIALS AND METHODS}

This is a cross-sectional study, conducted in dermatology clinics of Care institute of Medical Sciences, Banjara Hills, Hyderabad after approval of Institutional Ethics committee. The data was collected from 80 patients, 40 each of psoriasis and lichen planus who attended our OPD according to the inclusion and exclusion criteria, duration of study was 2 years. Inclusion criteria were set as all patients clinically diagnosed as psoriasis or lichen planus, clinically difficult cases or where diagnosis is doubtful are also included, all morphological variants of psoriasis and lichen planus were included, all patients with clinically doubtful findings, but with previous biopsy showing psoriasis and lichen planus. Exclusion criteria includes patients with prior clinical or histopathological evidence other than psoriasis or lichen planus, patients on topical or oral corticosteroids for treatment of psoriasis or lichen planus, one month prior to performing dermoscopy, psoriasis and lichen planus lesions with secondary infection, erythrodermic psoriasis.

The demographic data such as name, age, sex, chief complaints and other history of each case was noted. The diagnosis of psoriasis and lichen planus was made on the basis of standard definitions and clinical features, accepted in literature. Dermoscopic image capturing was performed by a single practitioner to avoid diversification during the procedure. Dermoscopic evaluation was performed by two independent investigators, who were unaware of the histopathological diagnosis. A hand held dermoscope (Hanse microscope-model HVS-CM 500PC), with charge coupled device, imaging sensor of 310 kilo pixels, offering a pixel resolution of $640 \times 480$ pixels, with video capability of 30 frames per second and magnification of 20 to 75 factors with automatic gain and exposure control. Digital dermoscopic images of lesions were obtained.

\section{Statistical Analysis}

For all the statistical analysis SPSS statistical software, version 17.0 for windows (SPSS Inc. Chicago, IL, USA) was used. ChiSquare $\left(\chi^{2}\right)$ test was done for statistical significance. In all instances, a " $p$ " value of 0.05 was considered significant. Charts were prepared with Microsoft Excel 2010 version.

\section{RESULTS AND DISCUSSION}

A total of 80 patients were taken into our study, 40 patients each with psoriasis and lichen planus. In psoriasis group, maximum number of patients were male $27(67.5 \%)$ and remaining were female $13(32.5 \%)$ with mean age group of 37.63 years. In lichen planus group, maximum number were female patients $21(52.5 \%)$, and the remaining were male $19(47.5 \%)$ with mean age group 34.40 years.

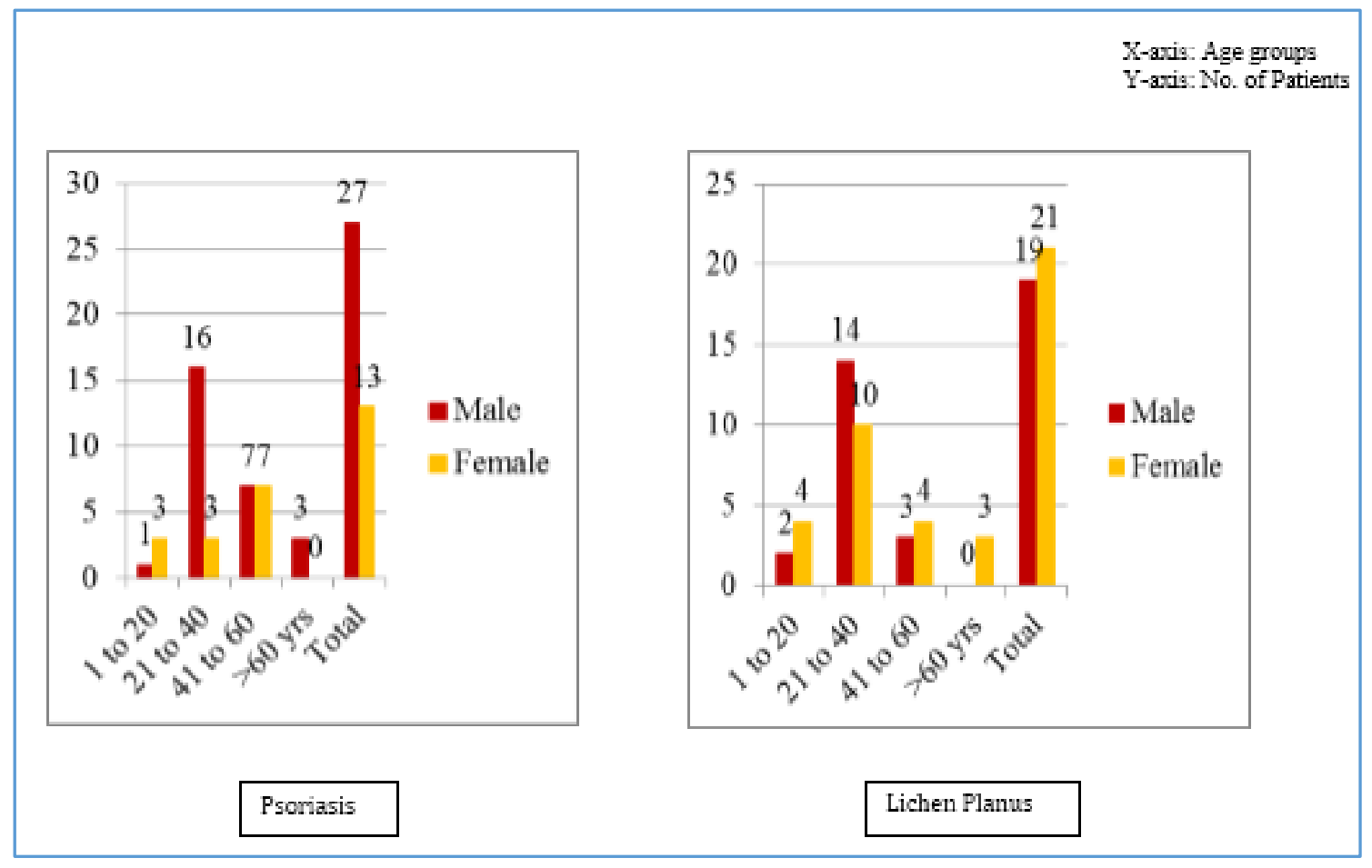

Fig. 1: Bar chart showing distribution of age as per gender in Psoriasis and Lichen planus

Out of 40 patients of psoriasis, the different morphological variants included were plaque psoriasis 25(62.5\%), guttate psoriasis $5(12.5 \%)$, hypertrophic psoriasis $5(12.5 \%)$, palmar psoriasis $3(7.5 \%)$, palmoplantar psoriasis $1(2.5 \%)$, and plantar psoriasis 1 (2.5\%). Out of 40 lichen planus cases, hypertrophic LP 12 (40\%), eruptive LP 9(22.5\%), annular LP 6(15\%), linear LP 5(12.5\%), follicular LP 3 (7.5\%), generalised LP 3 (7.5\%) and actinic LP 2 (5\%). 


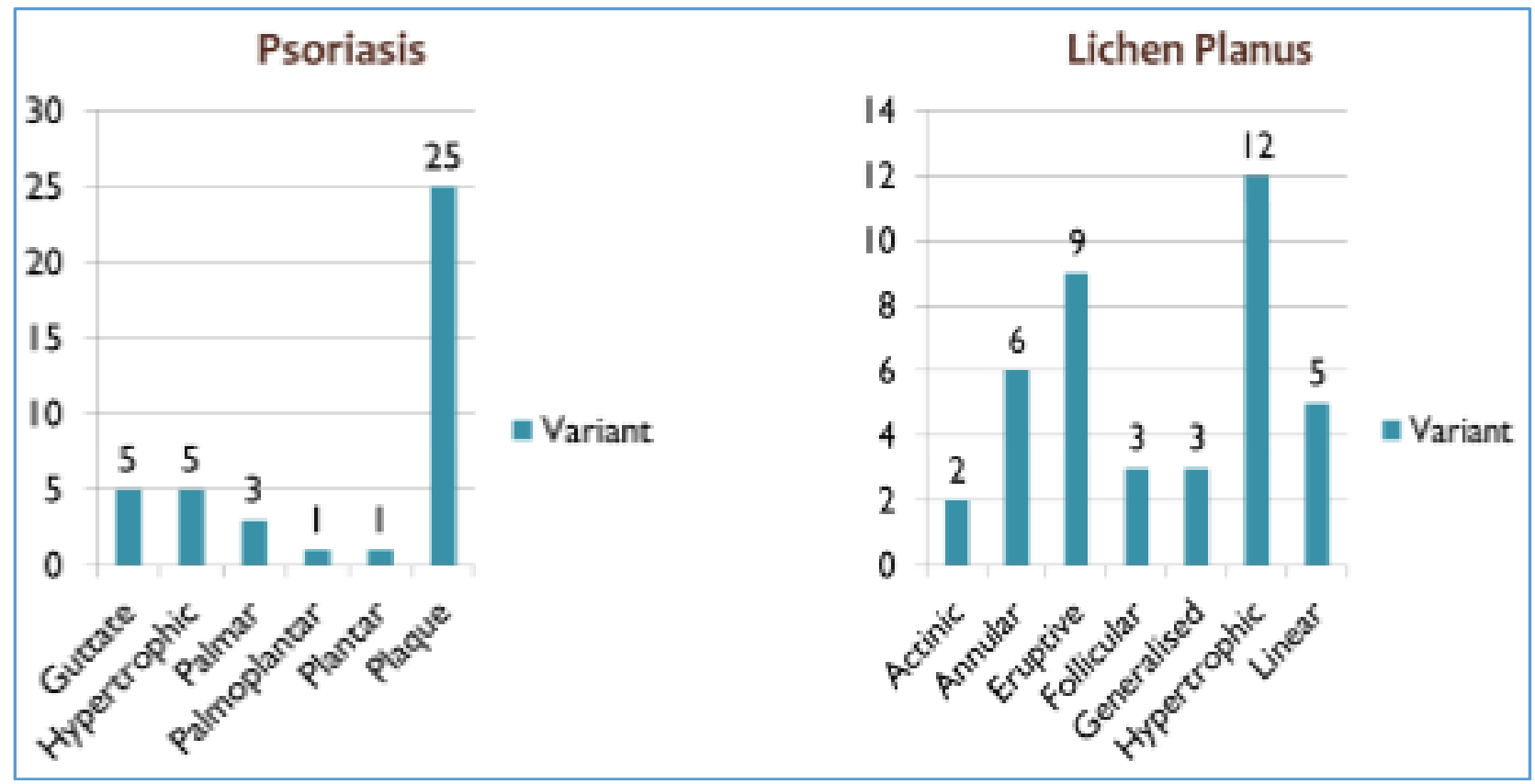

Fig. 2: Bar chart showing distribution of Morphologic variants of Psoriasis and Lichen Planus

The dermoscopic findings in psoriasis cases, maximum showed red globules 27(67.5\%) followed by glomeruli like vessels $10(25 \%)$. Background colour of light red was seen in $17(42.5 \%)$. Grey blue background was noted in $10(40 \%)$. Scales in psoriasis cases on dermoscopy were note as silvery white in maximum 35 (87.5\%) cases, greasy yellow in 3(7.5\%), grey blue in $2(5 \%)$ cases. Red globules were observed in $27(67.5 \%)$ cases Next important finding was glomeruli like vessels seen in $10(40 \%)$ patients. The only non-vascular finding observed was grey-blue dots, found in $3(7.5 \%)$ cases.
The dermoscopic findings in Lichen Planus cases, maximum were Wickham's striae in $24(60 \%)$ and grey-blue dots in $17(42.5 \%)$ cases. Grey blue background colour was noted in 32(45\%). Scales on dermoscopy were seen in 10 cases and clinical scales were noted in only 5 cases. Wickham's striae was the most common non-vascular finding noted in 24 cases of LP. Grey blue dots were seen in 17 cases. Yellow dots noted in 6 cases. Comedo like openings and corn pearl was seen in 1 cases each. One case showed red globules and glomeruli like vessels.

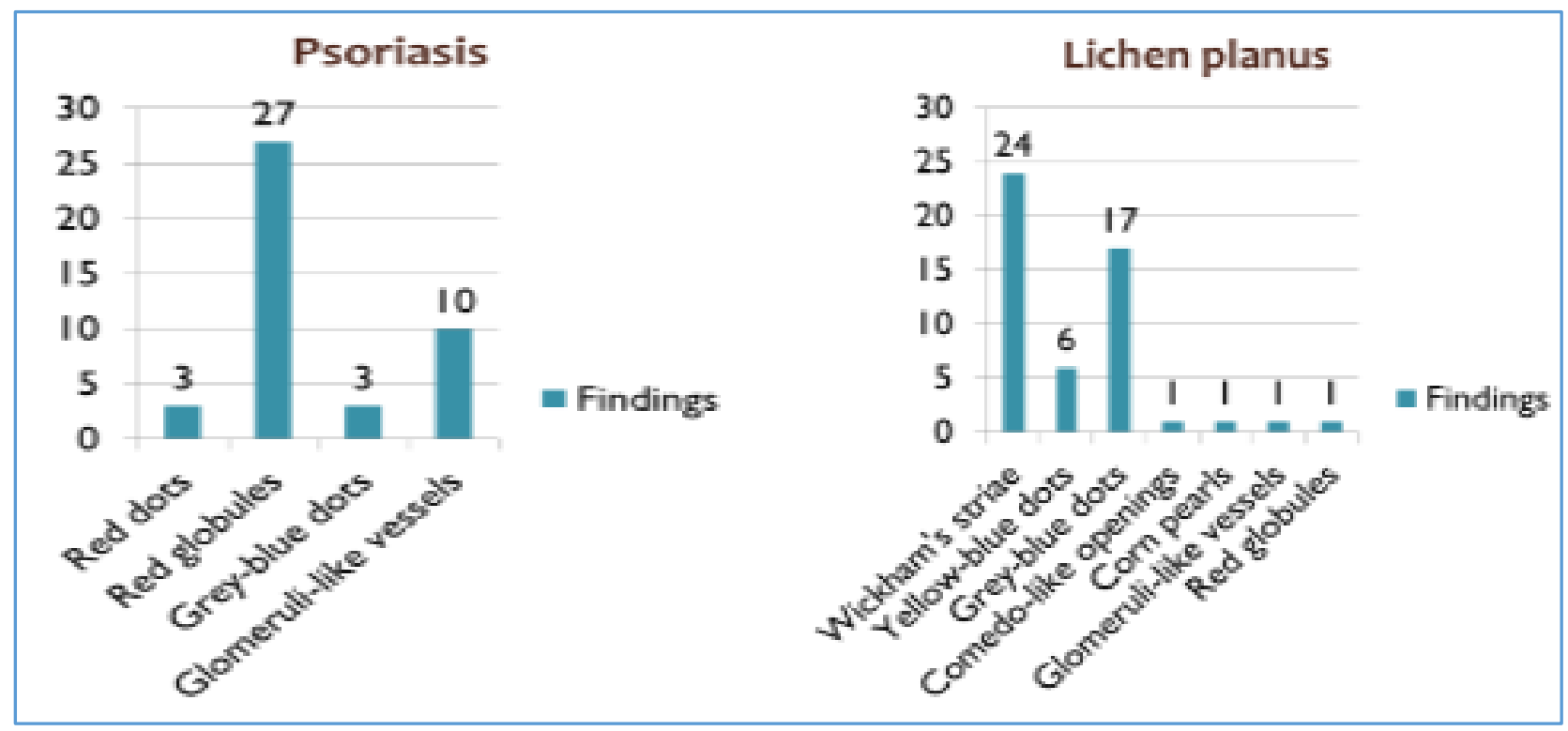

Fig. 3: Bar chart showing dermoscopic findings of Psoriasis and Lichen Planus

Histopathological examination was done in total of $14(35 \%)$ psoriasis cases, out of which 11(27.5\%) were dermoscopically consistent with psoriasis and remaining $3(7.5 \%)$ were dermoscopically not consistent with psoriasis.

Histopathological examination was done in total of $23(57.5 \%)$ lichen planus cases, out of which $15(37.5 \%)$ were dermoscopically consistent with LP and 8(20\%) cases were dermoscopically not consistent with LP. 


\begin{tabular}{|c|c|c|c|}
\hline $\begin{array}{c}\text { Dermoscopic } \\
\text { Finding }\end{array}$ & $\begin{array}{c}\text { Psoriasis } \\
\text { (n=40) } \\
\text { N (\%) }\end{array}$ & $\begin{array}{c}\text { Lichen } \\
\text { Planus } \\
\text { (n=40) } \\
\text { N (\%) }\end{array}$ & P-value \\
\hline Wikham's striae & 0 & $24(60)$ & 0.0001 \\
\hline Yellow brown dots & 0 & $6(15)$ & 0.11 \\
\hline Gray blue dots & $3(7.5)$ & $17(42.5)$ & $<0.0001$ \\
\hline $\begin{array}{c}\text { Comedo like } \\
\text { openings }\end{array}$ & 0 & $1(2.5)$ & 0.314 \\
\hline Corn pearls & 0 & $1(2.5)$ & 0.314 \\
\hline Red dots & $3(7.5)$ & 0 & 0.077 \\
\hline Red globules & $27(67.5)$ & $1(2.5)$ & 0.001 \\
\hline $\begin{array}{c}\text { Glomeruli like } \\
\text { vessels }\end{array}$ & $10(25)$ & $1(2.5)$ & 0.003 \\
\hline $\begin{array}{c}\text { Light red } \\
\text { background }\end{array}$ & $17(42.5)$ & $4(10)$ & 0.001 \\
\hline $\begin{array}{c}\text { Gray blue } \\
\text { background }\end{array}$ & $10(40)$ & $32(80)$ & 0.034 \\
\hline \multicolumn{2}{|c|}{$35(87.5)$} & $10(40)$ & 0.057 \\
\hline \multicolumn{2}{|c|}{ Tables 1: Differences in the dermoscopic features of } \\
\hline \multicolumn{2}{|c|}{ Psoriasis and Lichen Planus } \\
\hline
\end{tabular}

As per statistical analysis, there is statistical significance at $5 \%$ confidence interval $(\mathrm{p}=0.001, \chi=37.143)$ of red globules in comparison of psoriasis and lichen planus. There is also a statistical significance at $5 \%$ confidence level $(\mathrm{p}=0.001$, $\chi=34.286)$ of Wickham's striae, grey blue dots ( $p=0.000$, $\chi=13.067)$ and glomeruli like vessels $(p=0.003, \chi=8.538)$ in comparison between psoriasis and lichen planus.

\section{DISCUSSION}

Our study suggests significant differences in the dermoscopic features of common inflammatory dermatosis psoriasis and lichen planus. In our study while comparing the dermoscopic findings of both psoriasis and lichen planus, vascular features were observed to be significantly high in psoriasis and nonvascular features were significantly high in lichen planus.

\begin{tabular}{|c|c|c|c|}
\hline $\begin{array}{c}\text { Dermoscopic Findings } \\
\text { in Psoriasis }\end{array}$ & $\begin{array}{c}\text { Vazquez-Lopez, Jose } \\
\text { Antonio et al. }^{\mathbf{}^{2}}\end{array}$ & $\begin{array}{c}\text { Yan Pan, Alex J. } \\
\text { Chamberlein et al. }^{\mathbf{6}}\end{array}$ & Present Study \\
\hline Red globules & $20 / 20(100 \%)$ & - & $27 / 40(67.5 \%)$ \\
\hline Glomeruli like vessels & - & $12 / 100(12 \%)$ & $10 / 40(25 \%)$ \\
\hline Red dots & 0 & $100 / 100(100 \%)$ & $3 / 40(7.5 \%)$ \\
\hline Light red background & - & $78 / 100(78 \%)$ & $17 / 40(42.5 \%)$ \\
\hline \multicolumn{2}{|r|}{ Table 2: Comparison of dermoscopic features of Psoriasis with previous studies } \\
\hline
\end{tabular}

\begin{tabular}{|c|c|c|}
\hline Dermoscopic findings in Lichen Planus & Vazquez-Lopez et al. $^{.}$ & Present Study $^{-1}$ \\
\hline Wickham's striae & $23 / 25(92 \%)$ & $24 / 40(60 \%)$ \\
\hline Gray-blue dots & $5 / 25(20 \%)$ & $17 / 40(42.5 \%)$ \\
\hline Yellow-brown dots & $5 / 25(20 \%)$ & $6 / 40(16 \%)$ \\
\hline Comedo-like openings & $4 / 25(16 \%)$ & $1 / 40(2.5 \%)$ \\
\hline Corn pearls & $3 / 25(12 \%)$ & $1 / 40(2.5 \%)$ \\
\hline Milium cysts & $1 / 25(4 \%)$ & 0 \\
\hline Table 3: Comparison of dermoscopic features of Lichen Planus with previous studies \\
\hline
\end{tabular}

The most significant vascular finding associated with psoriasis was red globules 27/40 (67.5\%) followed by glomeruli like vessels 10/40 (25\%) and red dots $3 / 40$ (7.5\%). Red globules on histology correlated with dilated and tortuous capillary loops, glomeruli like vessels with irregular tortuous capillaries and red dots with normal papillary vessels. In a similar study by Vazquez-Lopez, Jose Antonio et al, red globules were seen in all the patients with psoriasis 20/20 $(100 \%) .^{3}$ Similarly in another study conducted by VazquezLopez and J. Kreusch et al. 414 consecutive patients with nontumoral dermatosis were evaluated, of which 40 patients had psoriasis and red globules were seen in all 40 of them (100\%). ${ }^{4}$ In study done by Yan Pal, Alex J. Chamberlain et al to distinguish psoriasis from skin carcinomas, red globules were observed in only 32 out of 100 cases (32\%). ${ }^{6}$ Glomeruli like vessels were seen in 12/100 cases (12\%) in a study conducted by Yan Pan, Alex J. Chamberlein et al. ${ }^{6}$

However, no other study had documented GLV as significant finding. This difference is likely due to result of variable terminologies used in studies. Red dots were noted in $4 / 20(20 \%)$ in a study by Vazquez-Lopez, Jose Antonio et al while Vazquez-Lopez and J. Kreusch et al study found no red dots. ${ }^{3,4}$ However interestingly Yan Pal, Alex J. Chamberlain et al study showed red dots in all the patients 100/100(100\%). ${ }^{6}$ In our study, light red background was observed to be maximum seen in $17 / 40(42.5 \%)$ cases of psoriasis while greyblue background noted in 10/40 (25\%). In study by Yan Pan,
Alex J. Chamberlein et al light background was observed in $78 / 100(78 \%)$ and in study by A. Lallas, A. Kyrgidis et al. it was seen in 34/83 (41\%) cases of psoriasis.6,8 In our study, greyblue background was observed in hypertrophic psoriasis cases. Grey-blue background was not observed in previous studies as they have not included various morphological variants into their studies.

In our study, scales were noted in all psoriasis cases. Silvery white scales was most commonly seen in $35 / 40$ $(87.5 \%)$ psoriasis cases, greasy yellow scales in 3/40 (7.5\%). In study by A. Lallas, A. Kyrgidis et al. white scales were noted in 58/83 (70\%) psoriasis cases. ${ }^{8}$

The most significant dermoscopic finding of lichen planus observed in our study was Wikham's striae noted in $24 / 40(60 \%)$. In a similar study by Vazquez-Lopez, Jose Antonio et al, Wikham's striae was observed in 23/25 (92\%) and in study by Wikham's striae were observed in $24 / 25$ (96\%) lichen planus cases. The results of Wikham's striae in our study were slightly less compared to their study because we have taken only clinically consistent cases, but in their study all biopsy proven cases were included. Wikham's striae on histology correlates with the increased granular layer.

The next common non-vascular finding in our study in lichen planus cases was grey-blue dots seen in 17/40 (42.5\%). In study by Vazquez-Lopez et al. it was seen in 2/25 (20\%) cases. Grey-blue dots correlates on histology with melanophages in dermis. 
The next finding in lichen planus cases was yellow blue dots in 6/40 (16\%) 925 in our study, which was comparable with the study conducted by Vazquez-Lopez et al 5/25(20\%). Yellow-blue dots on histology correlates with vacuolar changes in basal cells and spongiosis. Other non-vascular findings seen our study were comedo like openings in $1 / 40(2.5 \%)$, corn pearl in $1 / 40(2.5 \%)$, milium like cysts were not seen in our study. In study by Vazquez-Lopez et al.3,7 the findings were comedo like openings in $4 / 25$ (16\%), corn pearl in $3 / 25(12 \%)$ and milium cyst in $1 / 25(4 \%)$.

Vascular findings were not significant in lichen planus patients. In our study histopathological correlation was done prospectively to compare and confirm the dermoscopic findings, thereby having advantage of obviating the need for invasive biopsy in patients. While in previous studies all biopsy proven cases were included. The number of patients included in our study though slightly more than previous studies is still found to be small, especially while comparing rare findings.

\section{CONCLUSION}

In our study, almost all cases of psoriasis showed predominantly vascular findings. Among vascular findings we found red globules to be the most common finding. It is consistent with other previous studies done by Vazquez-Lopez et al.3,4,7 In lichen planus, non-vascular findings, mainly Wikham's striae was the most common finding seen. In our study, dermoscopy enhanced the demonstration of Wikham's striae which were not visible with the naked eye.7,9 After observing different dermoscopic findings in psoriasis and lichen planus we are able to diagnose both the diseases dermoscopically with consistent findings without the need for invasive skin biopsy. Our observations clearly showed that simultaneous evaluation of both vascular and non-vascular findings improves surface microscopy of inflammatory dermatosis. $^{3-9}$ In conclusion, dermoscopy is a valuable tool for improving the accuracy of differentiation of non-pigmented scaly lesions. It provides a quick, simple and non-invasive aid.

The major benefit from improved dermoscopic differentiation of these common scaly lesions is a reduction in need for a skin biopsy. In addition, dermoscopy also helps in monitoring the response to therapy. Finally we must take into account that clinical use of dermoscopy in inflammatory dermatosis not only improves the diagnostic ability but also improves other fundamental aspects of daily practice and addition of new easily recognizable images for visual teledermatology. It has a positive psychological placebo effect on patients suffering from common dermatosis being examined by means of dermoscopy instead of naked eye cannot be neglected. The definitions of dermoscopic findings of inflammatory scaly diseases should be standardized all over the world.

\section{LIMITATIONS}

Biopsy could not be done in all the cases due to lack of consent from the patients. In spite of dividing psoriasis and lichen planus into different morphological variants we could not correlate any specific dermoscopic findings with the morphological variants due to small sample size. These limitations should be assessed in the future studies.

\section{REFERENCES}

1. Nischal KC, Khopkar U. Dermoscope. Indian Journal of Dermatology, Venereology and Leprology. 2005;71(4):300-3.

2. William Stolz, Peter Bilek, Michael Landchaer, et al.; Color atlas of dermatoscopy, $1^{\text {st }}$ ed. Germany: Blackwell Publications. 1994; p.7-10.

3. Vazquez-Lopez F, Manjon-Haces JA, Maldonado-Seral C, Raya-Aguado C, Perez-Oliva N, Marghoob AA Dermoscopic features of plaque psoriasis and lichen planus: new observations. Dermatology. 2003;207(2):151-6.

4. Vázquez-López F, Kreusch J and Marghoob AA. "Dermoscopic semiology: further insights into vascular features by screening a large spectrum of non-tumoral skin lesions." British Journal of Dermatology 150.2 (2004):226-231.

5. Zalaudek I, Argenziano G, Di Stefani A, Ferrara G, Marghoob AA, Hofmann-Wellenhof R, et al. Dermoscopy in general dermatology.

Dermatology. 2006;212(1):7-18.

6. Pan Y, Chamberlain AJ, Bailey M, et al. Dermatoscopy aids in the diagnosis of the solitary red scaly patch or plaque - features distinguishing superficial basal cell carcinoma, intra-epidermal carcinoma and psoriasis. J Am Acad Dermatol 2008;59:268-74.

7. Vazquez Lopez F, Alvarez Cuesta C, Hidalgo Garcia Y, et al. The hand held dermatoscope improves the recognition of Wickham Striae and capillaries in lichen planus lesions. Arch Dermatol 2001;137:13-76.

8. Lallas A, Kyrgidis A, Tzellos TG, Apalla Z, Karakyriou E, Karatolias A, et al. Accuracy of dermoscopic criteria for the diagnosis of psoriasis, dermatitis, lichen planus and pityriasis rosea. The British journal of dermatology. 2012;166(6):1198-205.

9. Vazquez Lopez F, Palacious Garcia L, Gomez Diez S, et al. Dermoscopy of discriminating between lichenoid sarcoidosis and lichen planus. Arch Dermatol 2011;147:11-30.

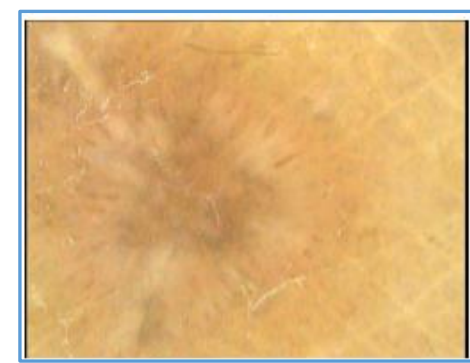

Wickham's Striae

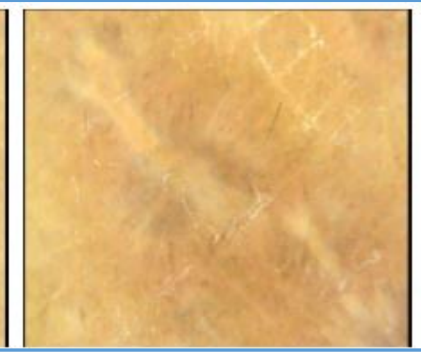

WS "brush like" (Micro-Koebners)

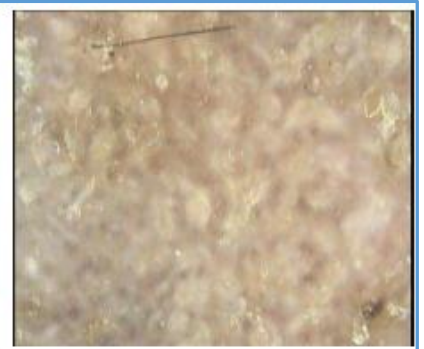

Grey-Blue Dots 


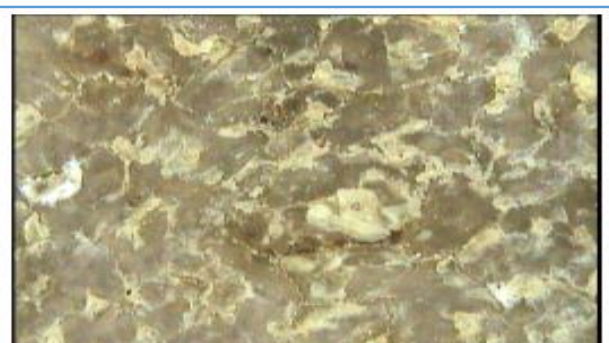

Comedo like Openings

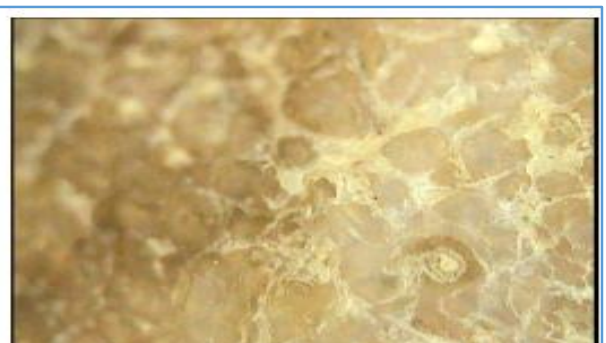

Corn Pearl

Fig. 4: Images showing dermoscopic features of Lichen Planus

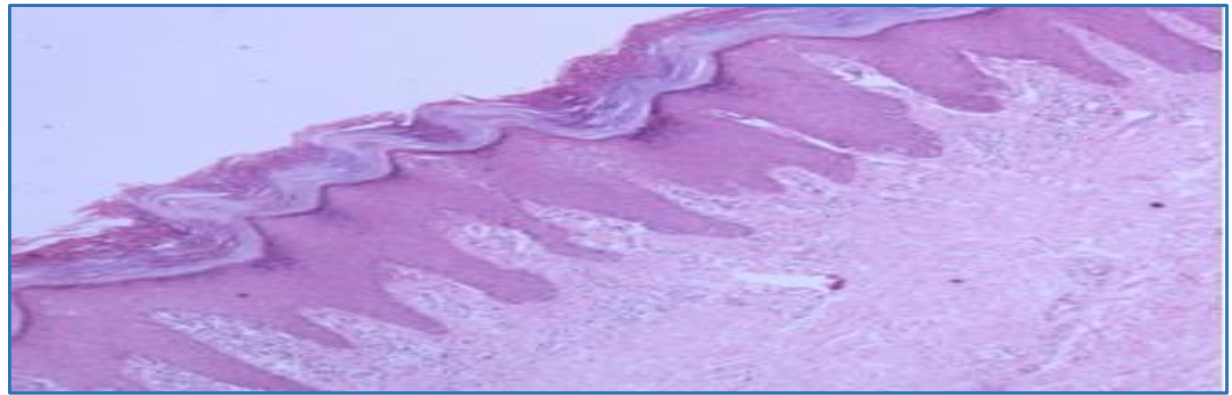

Fig. 5: Image showing Histopathological features of Lichen Planus

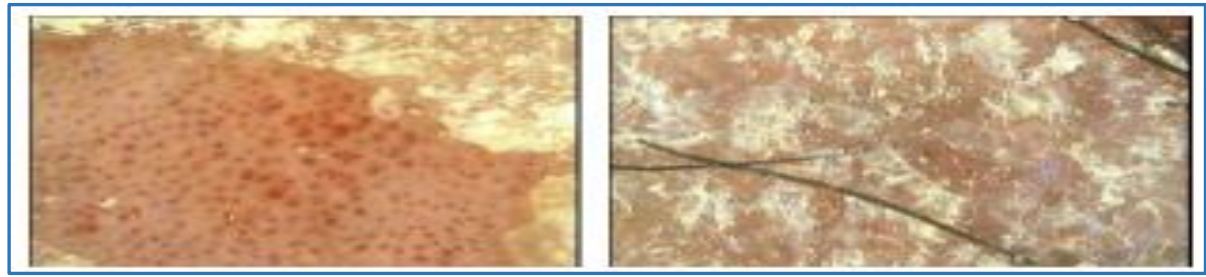

Red Globules and Red Dots Silvery White Scales

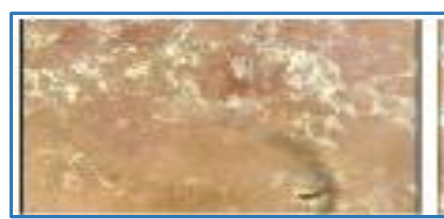

Light Red background

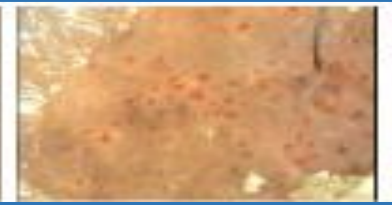

Glomeruli like vessels

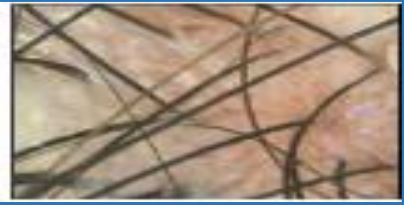

Red dots scalp psoriasis

Fig. 6: Images showing Dermoscopic features of Psoriasis

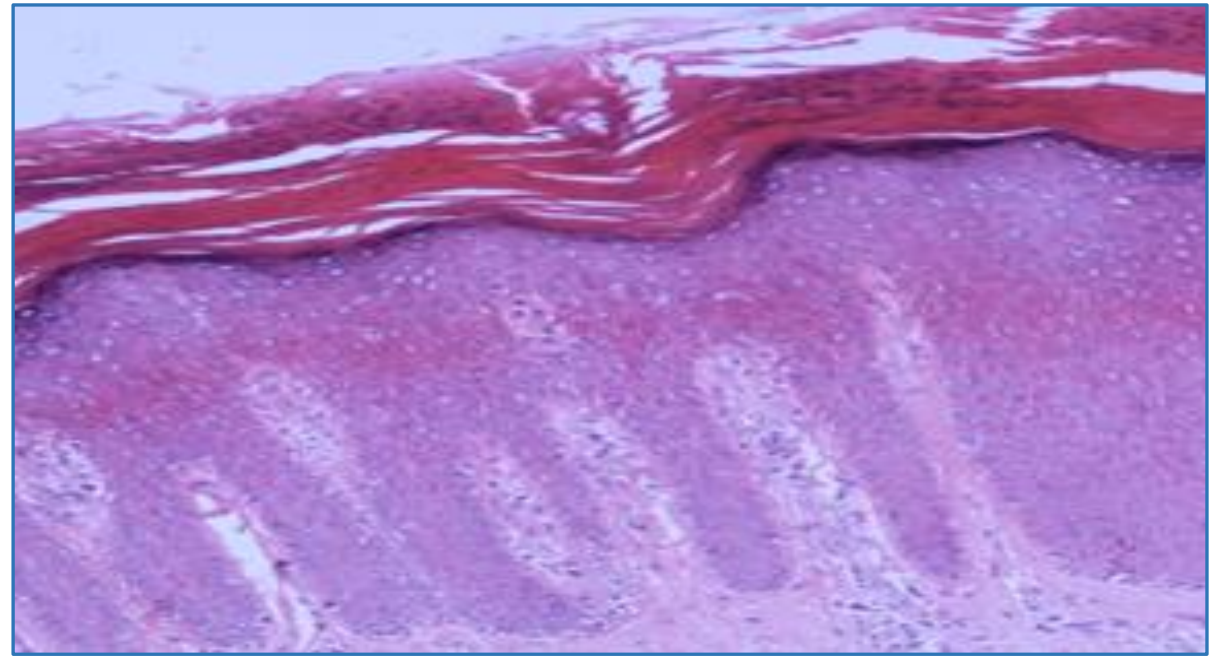

Fig. 7: Image showing Histopathological features of Psoriasis 